\title{
Protection des espaces agricoles et naturels : une analyse des outils américains et français
}

farmland and open space preservation: an analysis of u.s. vs. french tools

Jean-Christophe Dissart

\section{(2) OpenEdition}

\section{Édition électronique}

URL : http://journals.openedition.org/economierurale/578

DOI : 10.4000/economierurale.578

ISSN : 2105-2581

\section{Éditeur}

Société Française d'Économie Rurale (SFER)

\section{Édition imprimée}

Date de publication : 1 mars 2006

Pagination : 06-25

ISSN : 0013-0559

\section{Référence électronique}

Jean-Christophe Dissart, «Protection des espaces agricoles et naturels : une analyse des outils américains et français », Économie rurale [En ligne], 291 | Janvier-février 2006, mis en ligne le 05 janvier 2008, consulté le 19 avril 2019. URL : http://journals.openedition.org/economierurale/578 ; DOI :

10.4000/economierurale.578

(C) Tous droits réservés 


\title{
Protection des espaces agricoles et naturels: Une analyse des outils américains et français
}

\author{
Jean-Christophe DISSART* • Cemagref Grenoble, UR DTM \\ Courriel : jean-christophe.dissart@cemagref.fr
}

\section{Introduction}

$\mathbf{E}_{\mathrm{n}}^{\mathrm{n}}$ n raison d'une plus grande accessibilité physique (transport) et économique (coût du foncier), un espace périurbain s'est développé autour de la plupart des agglomérations d'Europe de l'Ouest et d'Amérique du Nord. Les enjeux liés à l'occupation de cet espace l'on rendu déterminant en matière d'aménagement, puisque s'y côtoient villes périphériques et centres commerciaux mais aussi exploitations agricoles et espaces naturels.

En particulier, la protection des espaces agricoles et naturels est importante pour des raisons de production alimentaire mais aussi d'entretien du paysage, ce dernier contribuant à la qualité de vie d'une agglomération.

Dans cet article, les espaces agricoles font référence aux terres cultivées et pâtures ; les espaces naturels comprennent les forêts, parcs et habitats naturels. De manière générale, les espaces agricoles et naturels correspondent aux espaces non bâtis ou aux sols non artificialisés. La plupart des techniques présentées peuvent servir à protéger indifféremment les espaces agricoles ou naturels, la principale distinction étant que les premiers demeurent des biens à usage privé.

L'analyse repose sur une comparaison des outils (tels que décrits dans la littérature et la législation) développés à la fois en France et aux États-Unis afin de protéger les espaces agricoles et naturels. En effet, les
États-Unis disposent de plus de foncier pour accommoder la croissance urbaine, mais, en raison de densités résidentielles et commerciales plus faibles, le mitage et la conversion de l'espace non bâti y sont plus importants. Pour protéger ces espaces, ils ont développé des outils qui sont intéressants car assez différents des outils français dans leur approche : la culture politique américaine accorde moins d'importance aux approches réglementaires et favorise le développement d'outils basés sur les mécanismes de marché.

Le reste de cet article est divisé en trois sections. Basée sur des statistiques nationales, la deuxième section montre le rythme de conversion des espaces agricoles en France et aux États-Unis, ainsi que les enjeux liés à la protection des espaces agricoles et naturels. Suite à une présentation des différences entre les systèmes d'aménagement français et américains, les principales mesures de protection développées par ces deux pays sont détaillées en fonction d'une typologie commune. La section finale conclut sur l'opportunité de compléter les outils français.

Les contributions de cette analyse résident à trois niveaux : proposition d'une typologie des mesures de protection des espaces non bâtis, présentation des outils américains (et français) en la matière, et recommandations en termes de politique publique. De manière générale, l'idée est moins de préserver chaque hectare que de ralentir le rythme de conversion de ces espaces. 
RECHERCHES

\section{Protéger les espaces agricoles et naturels ?}

\section{La perte d'espaces agricoles}

De manière générale, les tensions par rapport à l'utilisation du sol sont liées à plusieurs variables clés. Ces dernières, répertoriées dans le tableau 1 , montrent que la France est plus densément peuplée que les États-Unis, avec moins de terres agricoles par habitant. année $0,40 \%$ de leurs espaces agricoles, contre $0,26 \%$ en France (Agreste, 2004 ; NASS, 2004). Les valeurs absolues sont également impressionnantes : alors que la France a consommé «seulement » 78451 ha par an sur la même période de référence, en moyenne, les États-Unis ont converti 1546021 ha chaque année, soit la moitié de la surface de la Belgique ou presque deux fois la surface de l'Alsace.

Tableau 1. Statistiques comparatives

\begin{tabular}{lrr}
\hline Variable & France & États-Unis \\
\hline Population (estimation Juillet 2003) & 60180529 & 290342554 \\
Taux de croissance de la population (est. 2003), \% & 0,42 & 0,92 \\
Surface, $\mathrm{km}^{2}$ & 545630 & 9158960 \\
Densité de population, \#//km² & 110 & 32 \\
Urbanisation, \% & 76 & 77 \\
Surface des sols agricoles utilisés, \% & 35,41 & 19,54 \\
Surface des sols agricoles utilisés par personne, ha & 0,32 & 0,62 \\
PNB par habitant (parité de pouvoir d'achat, est. 2002), $€$ & 21580 & 30129 \\
\hline
\end{tabular}

Sources : tous chiffres de CIA (2004), à l'exception d'urbanisation (nationmaster.com)

Les deux pays présentent le même niveau d'urbanisation et des surfaces agricoles généreuses. Aux États-Unis, la surface totale est presque 17 fois plus importante qu'en France, mais la France présente une proportion plus importante de cette surface en sols agricoles utilisés (35 vs. $20 \%$ ). Avec un niveau de revenu plus élevé, associé à une forte consommation (y compris celle de foncier) et à une croissance de la population deux fois plus élevée qu'en France, les États-Unis connaissent une pression importante sur les ressources naturelles.

Ainsi, les espaces agricoles régressent ${ }^{1}$ à la fois en France et aux États-Unis mais beaucoup plus dans ces derniers. Sur la période 1992-1993 à 2001-2002, en moyenne, les États-Unis ont perdu chaque

1. Les statistiques sur les espaces naturels (open space) ne sont pas disponibles. Ces espaces recouvrent des utilisations variées du sol, ce qui complique les comparaisons internationales au cours du temps.
On peut ajouter que des sols agricoles sont également transformés en sols boisés, réduisant de fait la déprise au regard d'enjeux environnementaux, mais cela ne remet pas en cause l'artificialisation du territoire.

Compte tenu de ce rythme de conversion, il n'est pas surprenant que les ÉtatsUnis aient développé des outils de protection des espaces agricoles et naturels, de la même façon que les pays d'Europe de l'Ouest (la France en particulier) l'on fait en raison de surfaces moins importantes et de densités de population plus élevées.

\section{Pourquoi protéger les espaces agricoles et naturels}

Les espaces agricoles et naturels convertis sont en majorité voués à des utilisations bâties et à des réseaux de communication. Liés à un niveau de richesse et à une conscience environnementale renforcée, des arguments ont été avancés pour protéger ces espaces au titre d'enjeux économiques, esthétiques et environnementaux (AFT, 2003). 
Selon l'argument économique, les espaces agricoles devraient être protégés car il s'agit de maintenir la base du système agroalimentaire, important pour les économies française et américaine, à la fois en termes de balance commerciale, de création d'emploi et de revenu. Le maintien des espaces agricoles permet d'accompagner l'augmentation de la demande globale liée à l'augmentation de la population et des revenus et à l'ouverture des marchés. D'ailleurs, les espaces agricoles proches des zones métropolitaines sont parmi les plus productifs (et menacés) car, historiquement, les hommes se sont plutôt établis à proximité des terres agricoles les plus planes et fertiles.

Les espaces agricoles et naturels devraient également être protégés pour des raisons fiscales : un développement urbain éparpillé se traduit par des coûts d'équipement élevés qui sont financés par des impôts accrus ; de manière générale, les espaces naturels sont sources d'aménités qui augmentent les valeurs des propriétés et les revenus issus du tourisme (Brabec, 1994).

La valeur des espaces agricoles et naturels, toutefois, va au-delà de critères économiques : exploitations agricoles et paysages attrayants contribuent à la singularité d'une communauté, fournissant des repères dans l'espace et augmentant la qualité de vie locale.

Dans une perspective environnementale, les espaces agricoles et naturels fournissent aussi des services écologiques comme le maintien de la biodiversité, la protection des zones humides, le filtrage des eaux résiduaires, le rechargement des nappes phréatiques, la séquestration de carbone et une contribution à la qualité de l'air. Les sols devraient être économisés au titre de ressource finie et non renouvelable.

En revanche, des arguments ont également été avancés contre la protection des espaces agricoles (Gordon \& Richardson, 1998). D'abord, les niveaux de productivité actuels sont tels que la perte d'espace agricole ne constitue pas une menace pour l'offre alimentaire nationale. Ensuite, les subventions agricoles entraînent des distorsions de l'économie : l'agriculture ne crée pas autant d'emplois que les autres secteurs économiques, si bien que la protection de ce secteur se traduit par un gaspillage de ressources. Les critiques font également remarquer les coûts environnementaux de l'agriculture liés à la pollution diffuse. Enfin, en matière d'aménagement, il est reproché à la plupart des programmes de protection d'être coûteux et inefficaces parce qu'ils ne font que rediriger l'urbanisation et, dans certains cas, contribuent à l'étalement des zones bâties.

En conclusion, bien que l'offre alimentaire ne paraisse pas en danger et que l'activité agricole puisse avoir des impacts écologiques négatifs, il est prudent de s'intéresser à la protection des espaces agricoles, vu leur rythme de conversion actuel (Daniels, 1999a). De plus, des simulations montrent que la perte de foncier pourrait augmenter avec l'évolution de la démographie rurale (Cavailhès, 1995). Les préoccupations liées à la conversion des espaces agricoles et naturels sont de plus en plus fortes et une gamme d'outils a été développée pour les protéger.

\section{Présentation des outils américains et français}

\section{1. Éléments d'aménagement clés}

En France, comme aux États-Unis, la structure de protection des espaces agricoles et naturels est définie par des éléments d'aménagement fondamentaux, notamment par rapport au contenu du droit de propriété et à la place de l'État fédéral.

Dans les deux pays, le droit de propriété privée est l'un des principes du droit, mais le contenu de ce droit y est cependant très différent. En France, le droit de propriété sur le sol est basé sur une conception unitaire alors qu'aux États-Unis la propriété du 
RECHERCHES

sol est un faisceau de droits (bundle of rights) qui accompagne le foncier ${ }^{2}$. Ces droits sont séparables et peuvent concerner non seulement la terre, l'eau, le sous-sol (exploitation), l'air (survol) qui y sont rattachés, mais aussi le droit d'utiliser le foncier de plusieurs manières : don, vente, location, construction, exclusion du public ou legs (Daniels \& Bowers, 1997). En particulier, le droit de construction peut être affecté par une servitude de protection (conservation easement) qui le restreint ou l'élimine.

Le droit de propriété privée est protégé par la constitution américaine et le $5^{\circ}$ amendement exige que le gouvernement paie une « juste indemnisation » à un propriétaire en cas de « saisie » (taking). Toutefois, ce qui est considéré comme saisie est sujet à débat et ce sont les tribunaux des États ou la Cour Suprême qui décident si une réglementation est « allée trop loin» et a résulté en une prise de possession par le gouvernement. Le $14^{\mathrm{e}}$ amendement contient également une provision importante : les américains ont le droit de voyager librement, ce qui signifie qu'une collectivité locale ou un État ne peut imposer de limite de population.

Contrairement aux États-Unis, il existe en France un principe général de non-indemnisation des servitudes d'urbanisme (Châteaureynaud, 2003 ; Renard, 1999). Ainsi, les aménageurs français n'ont pas à affronter la question des saisies lorsqu'ils soumettent une parcelle privée à un usage spécifique tel que le patrimoine naturel. En d'autres termes, le droit de propriété privée ne peut être violé arbitrairement (justification par l'intérêt général), mais son

\footnotetext{
2. Moins souple que le système américain, le droit français connaît toutefois le démembrement de propriété qui consiste à séparer les différents droits composant le droit de propriété (usus, fructus et abusus) entre plusieurs personnes.
}

infraction ne débouche pas sur une indemnisation ${ }^{3}$.

D'autre part, en France, et par opposition à d'autres pays européens comme l'Italie ou l'Espagne, la responsabilité de l'aménagement a été principalement allouée au gouvernement central : les lois adoptées par le pouvoir exécutif encadrent la mise en œuvre des politiques spatiales qui peuvent être déléguées aux collectivités locales si elles sont dotées d'un plan d'urbanisme (Caillaud \& Aubert, 2003). D'autre part, ces compétences sont encadrées par des normes supérieures et sujettes au contrôle de légalité exercé par le préfet (Caillaud \& Aubert, 2003 ; Cullingworth, 1994). Ce système comprend donc des recours légaux pour les acteurs du processus d'aménagement. Enfin, les moyens de l'État s'articulent non seulement autour d'outils réglementaires mais aussi d'outils financiers, y compris des fonds structurels de l'Union européenne (Casteigts, 2002).

Aux États-Unis, la constitution a alloué la responsabilité de l'utilisation du sol aux gouvernements des États, de sorte que le gouvernement fédéral est peu impliqué dans les questions de planification spatiale (Alterman, 1997). Le gouvernement central influence le foncier et l'activité agricoles principalement par des programmes d'aide financière et de classification des sols (Beesley, 1999). De plus, avec 50 États et de nombreux gouvernements locaux, les politiques de protection du foncier sont caractérisées par la variété, l'inventivité et l'expérimentation, et seules les politiques locales ayant réussi bénéficient d'une reconnaissance nationale (Alterman, 1997). Par conséquent, les mesures de protection

3. En France, des servitudes conventionnelles peuvent être instituées entre un propriétaire privé et certains organismes comme le Conservatoire du littoral où l'interdiction de bâtir qui pèse sur le terrain fait l'objet d'une indemnisation, mais ces outils ne sont pas fréquents. 
s'y présentent comme un ensemble d'outils transversaux par rapport aux politiques fonctionnelles, appliquant des contrôles sur l'usage des sols et une variété de mécanismes pour réguler leur conversion.

\section{Une typologie des techniques de protection des espaces agricoles et naturels}

Il existe plusieurs classifications des techniques de protection des espaces agricoles et naturels (Adelaja \& Schilling, 1999 ; AFT, 2002 ; AFT, 1997 ; Beesley, 1999). La classification proposée ici s'inspire de Daniels (1999b) qui la présente comme un ensemble de techniques de gestion de la croissance dans les espaces périurbains.

Le tableau 2 résume les outils existants aux États-Unis et en France en matière de protection des espaces agricoles et naturels. Pour chacun des types, seuls les outils les plus utilisés sont détaillés ci-après. Cette limitation est justifiée du fait que des revues plus exhaustives existent (pour les États-Unis, voir, par exemple : AFT, 1997 ; Daniels, 1999b ; Daniels \& Bowers, 1997) et que ces outils sont les plus fréquemment répertoriés.
Les directives de développement visent à guider l'aménagement à l'échelle d'une ou de plusieurs localités, aussi bien en termes de construction et d'équipement que de protection de l'environnement. Ensuite, le zonage définit les usages du terrain permis dans des zones spécifiques délimitées, en principe, à l'échelle communale. Les acquisitions de propriété font référence à la fois à la possibilité d'acquisition physique des terrains et la possibilité d'acquérir, de vendre ou d'échanger des droits à bâtir. Enfin, les incitations comportent des dispositions fiscales et juridiques qui renforcent l'intérêt de maintenir des terrains non bâtis.

\section{Outils américains de protection des espaces agricoles et naturels}

Certaines techniques sont mises en œuvre essentiellement au niveau des États, d'autres au niveau local, ces dernières venant souvent en renfort des premières (AFT, 2002). La description des outils s'inspire principalement d'AFT (1997) et de Daniels \& Bowers (1997).

Sous le registre des directives de développement on trouve principalement le master (ou comprehensive) plan (schéma directeur) et l'urban growth boundary (limite de croissance urbaine).

Tableau 2. Principales techniques de protection des espaces agricoles et naturels

\begin{tabular}{|c|c|c|}
\hline Type d'outil & États-Unis & France \\
\hline Directive de développement & $\begin{array}{l}\text { Master plan } \\
\text { Urban growth boundary }\end{array}$ & $\begin{array}{l}\text { Schéma de cohérence territoriale } \\
\text { Plan local d'urbanisme }\end{array}$ \\
\hline Zonage & $\begin{array}{l}\text { Agricultural protection zoning } \\
\text { Cluster zoning }\end{array}$ & $\begin{array}{l}\text { Règle de constructibilité limitée } \\
\text { Zones }(\mathrm{A}) \text { et }(\mathrm{N}) \text { des PLU } \\
\text { Zone agricole protégée }\end{array}$ \\
\hline Acquisition de propriété & $\begin{array}{l}\text { Purchase of development right } \\
\text { Transfer of development right } \\
\text { Private land trust }\end{array}$ & $\begin{array}{l}\text { Transfert de COS } \\
\text { Espace naturel sensible } \\
\text { Etablissement public foncier local } \\
\text { Zone d'aménagement différé } \\
\text { Conservatoire du littoral } \\
\text { SAFER }\end{array}$ \\
\hline Incitation & $\begin{array}{l}\text { Circuit breaker tax relief credit } \\
\text { Current use valuation } \\
\text { Agricultural district } \\
\text { Right-to-farm law }\end{array}$ & $\begin{array}{l}\text { Taxe sur le foncier non bâti } \\
\text { CTE/CAD }\end{array}$ \\
\hline
\end{tabular}

Note : afin que la traduction ne trahisse pas le sens original, les outils américains sont listés en langue anglaise 
RECHERCHES

Le but du master plan est de définir une vision et de guider le développement sur la base de projections de population et des besoins fonciers correspondants. Les avantages de ce plan, établi au niveau de la commune ou du comté, sont nombreux : donne une base légale au zonage et autres règles d'usage du sol, définit des objectifs de croissance et de protection, identifie des zones ciblées pour une gamme d'utilisations du sol, promeut le développement ordonné des équipements. Ses inconvénients principaux sont qu'il n'est pas légalement contraignant par lui-même, n'a pas à promouvoir de vision régionale, et peut être ignoré dans les décisions quotidiennes. Le master plan peut promouvoir la protection des espaces agricoles et naturels en encourageant la définition de limites de croissance urbaine ou l'adoption d'une zone agricole protégée ou encore en incorporant l'utilisation de programmes d'achat ou de transfert de droit à bâtir.

Mise en place par un État, une limite de croissance urbaine a pour objectif de gérer l'extension urbaine en contrôlant son timing et en déterminant les usages du sol permis aux niveaux local et régional. Ligne théorique tracée autour d'une agglomération, cette limite définit une zone permettant d'accommoder la croissance anticipée à 10-20 ans et limite l'extension des équipements. Cette mesure demande également aux collectivités d'identifier les sols à forte valeur de ressource et de les protéger de l'artificialisation. En conséquence, elle promeut un développement plus compact, moins coûteux en termes de service, décourage l'étalement urbain et peut protéger les espaces agricoles et naturels si elle est combinée à d'autres techniques. Cependant, elle nécessite un zonage restrictif au-delà de la limite et une politique de phasage de la croissance à l'intérieur. Si elle sous-estime les besoins, elle peut aussi entraîner une augmentation du coût du foncier liée à la restriction de l'offre. Enfin, un accord entre communes et comtés sur son tracé peut être difficile à obtenir.
Le zonage comprend principalement deux techniques : la délimitation d'une zone agricole protégée (agricultural protection zoning) et le zonage en grappe (cluster zoning).

Principalement mise en œuvre au niveau local, une zone agricole protégée identifie une ou plusieurs zones où l'agriculture est l'usage privilégié du sol et décourage (sinon interdit) d'autres usages. Le but est de limiter les conflits d'usage en séparant les activités agricoles des autres et de protéger une masse critique d'exploitations et de terres agricoles. L'identification de la zone est généralement basée sur des critères de localisation et de qualité du sol. Ce type de zonage peut aussi spécifier le nombre d'habitations par $\mathrm{km}^{2}$, la taille minimale des terrains $^{4}$ ou autoriser des activités commerciales (vente directe). Au-delà de son impact sur l'activité agricole, ce zonage permet aussi de maintenir l'espace non bâti et de limiter la spéculation immobilière, le tout à un coût réduit pour le contribuable. Cette technique, toutefois, requiert des terrains importants (ou nombreux) et contigus et des éléments sur la taille minimale des lots. De plus, la zone ciblée peut être parsemée de propriétés ; les propriétaires fonciers ne sont pas indemnisés pour la restriction d'usage ; les collectivités peuvent changer le zonage ; et les terrains sont vulnérables à l'annexion.

Également mis en œuvre au niveau local, le zonage en grappes permet ou exige que les bâtiments soient groupés sur des terrains dont la taille minimale est importante. Ce zonage est parfois utilisé, aussi, pour accorder aux promoteurs un bonus de densité sur un site tandis que la portion du terrain qui n'est pas bâtie est sujette à une servitude de protection. Le zonage en grappes permet un développement moins coûteux que le mode périurbain classique et plus

4. Les densités maximales vont de 0,4 (dans l'Ouest) à 12,3 (dans l'Est) habitations par $\mathrm{km}^{2}$. La taille minimale des terrains est de 16,2 ha et plus. 
sensible à la protection de l'environnement. Les détracteurs de cette technique soulignent qu'elle protège davantage le foncier que l'activité agricole parce que les propriétaires du terrain protégé peuvent ne pas vouloir louer leur propriété en raison des nuisances potentiellement associées à l'agriculture. Le zonage en grappes peut également se traduire par un étalement groupé (clustered sprawl).

Il existe trois principaux outils d'acquisition de propriété pour la protection des espaces agricoles et naturels : le programme d'achat de droit à bâtir (purchase of development right), le programme de transfert de droit à bâtir (transfer of development right) et la fiducie foncière privée (private land trust), qui tous reposent sur un droit à bâtir négociable. D'autres techniques existent, comme l'achat de propriété traditionnel (qui porte sur l'ensemble du faisceau de droits) ou l'expropriation (eminent domain).

Essentiellement mis en œuvre par les États, le programme d'achat de droit à bâtir consiste à payer les propriétaires fonciers pour qu'ils ne construisent pas sur leur terrain, ce qui offre une protection plus forte et plus durable que le zonage. Un propriétaire vend le droit à bâtir à une agence gouvernementale ou une organisation de conservation privée, qui en général lui paie la différence entre la valeur du foncier non bâti : la valeur de la servitude et celle constructible. Cette dernière correspond à l'usage « le meilleur et le plus élevé » (highest and best use), en général résidentiel ou commercial. Ainsi, l'acheteur acquiert la responsabilité d'imposer la servitude de protection tandis que le vendeur est indemnisé pour la perte du droit à bâtir. Les États peuvent jouer plusieurs rôles dans l'exécution de ce type de programme, dont le partenariat avec les collectivités locales pour acheter des servitudes.

Le programme d'achat de droit à bâtir est intéressant pour les propriétaires et la société. Pour les propriétaires, la technique capte la plus-value du terrain et l'indemnité peut servir à rembourser des emprunts ou réaliser des investissements. La valeur plus faible du terrain devenu non constructible facilite la reprise des exploitations ou les nouvelles installations. De plus, les propriétaires peuvent recevoir des crédits d'impôt et restreindre l'accès à la propriété qui demeure privée. Par rapport à la société, cet outil permet une protection permanente, aide à maintenir une masse critique de foncier non bâti et évite la question du taking. Les collectivités peuvent aussi cibler les terrains. Cependant, ce programme peut s'avérer coûteux pour une commune, avec opposition possible des contribuables. Il peut ainsi être difficile de protéger une surface significative et le programme peut résulter en un archipel de terrains protégés. Une diversité de moyens existe pour lever les fonds nécessaires, y compris dons privés, contributions croisées des collectivités et taxation locale.

Généralement établi au niveau local, un programme de transfert de droit à bâtir est utilisé pour déplacer le développement d'une zone agricole ou naturelle vers une zone de croissance plus proche des équipements. Le droit de construction est transféré d'une zone émettrice vers une zone réceptrice, de sorte qu'une servitude de protection permanente restreint l'usage de la zone émettrice tandis que la zone réceptrice peut être bâtie à une densité plus élevée que celle normalement permise par le zonage. Le gouvernement peut assumer plusieurs rôles : soit les collectivités approuvent les transactions entre propriétaires privés et promoteurs et contrôlent les servitudes ; soit les collectivités créent des banques de transfert qui achètent les droits à bâtir des propriétaires et les revendent à des promoteurs souhaitant construire à des densités plus élevées (Daniels \& Bowers, 1997).

Ainsi, mettre en place un programme de transfert requiert : 1) l'identification d'une zone à protéger, 2) l'identification d'une 
RECHERCHES

zone de croissance, 3 ) un ensemble de droits à bâtir, et 4) une procédure par laquelle les droits de construction sont transférés d'une propriété à l'autre (Daniels \& Bowers, 1997). Un programme de transfert offre tous les avantages d'un programme d'achat. De plus, il est davantage guidé par le marché et peut ne pas requérir de fonds publics. Ce programme est flexible, pouvant servir à protéger des terres agricoles aussi bien que des zones écologiquement sensibles ou des monuments historiques. La densité plus élevée dans les zones réceptrices permet d'utiliser les équipements publics à pleine capacité et d'assumer plus facilement une part équitable d'habitat social (Daniels, 1999b).

Bien que théoriquement efficace, la technique de transfert n'a pas été beaucoup utilisée en raison de sa complexité qui réside à plusieurs niveaux : éduquer et susciter l'intérêt des promoteurs et des propriétaires, identifier et s'entendre sur les zones émettrices et réceptrices, déterminer la valeur des droits à bâtir, mettre en place une banque de crédits de transfert et disposer d'un robuste marché de l'immobilier. Les propriétaires voisins de la zone réceptrice peuvent aussi s'opposer à une densité plus élevée (phénomène NIMBY). Enfin, les crédits de transfert sont généralement basés sur la surface possédée et pas nécessairement sur la localisation, la qualité des sols ou l'accès aux équipements (Daniels, 1999b).

Une fiducie foncière est une organisation de conservation privée, à but non lucratif, créée pour protéger les ressources naturelles (ainsi que les monuments historiques) pour le public. Une fiducie foncière achète ou accepte des dons de servitudes de protection, d'argent ou de propriété. Elle a aussi pour mission d'éduquer le public et peut conseiller les collectivités et particuliers en matière de planification immobilière. Le donateur de servitude peut recevoir un crédit d'impôt ; le bénéficiaire de la servitude doit la faire respecter. La plupart des servitudes sont permanentes, mais l'accord peut concerner un nombre d'années limité. Les avantages de cet outil sont multiples : protection permanente du foncier, possibilité de forger des partenariats publics-privés à faible coût pour la collectivité, déductions fiscales pour les donateurs alors que le terrain demeure en propriété privée, enfin le terrain reste dans l'assiette fiscale. Les inconvénients incluent le manque de fonds, la possibilité de créer des îlots de protection dispersés, peu de contrôle des collectivités sur la désignation des zones à protéger et des incitations fiscales insuffisantes pour nombre de propriétaires. Toutefois, Daniels (1999b) estime que les fiducies foncières sont des outils prometteurs en zone périurbaine.

Enfin, les techniques d'incitation pour la protection des espaces agricoles et naturels comprennent principalement la taxation préférentielle sur la propriété (property preferential taxation), le district agricole (agricultural district) et la loi sur le droit-à-exploiter (right-to-farm law).

La taxation préférentielle sert à encourager les propriétaires à maintenir le foncier en usage agricole ou naturel. Cet outil repose sur l'hypothèse que des impôts plus élevés réduisent les profits et que le manque de rentabilité est un facteur majeur de la conversion des terrains. Deux types de programme existent : 1) circuit breaker tax relief credit, qui offre un crédit d'impôt lorsque la taxe sur la propriété est supérieure à un pourcentage donné du revenu de l'exploitant ; 2) évaluation à l'usage courant (current use valuation), qui exige des collectivités qu'elles estiment le terrain agricole à sa valeur d'usage (non bâti) et non sa valeur (constructible) sur le marché. La technique peut effectivement encourager les propriétaires à garder leur terrain en usage non bâti, mais est critiquée pour ses règles d'éligibilité souvent laxistes. De plus, elle peut être utilisée par les spéculateurs fonciers ou les exploitants à temps partiel et peut se traduire par un manque à gagner significatif pour les collectivités. 
Autorisé par un État et appliqué localement, un district agricole est une zone créée pour une durée fixe et renouvelable, où l'activité agricole est encouragée par les avantages procurés aux propriétaires. Ces avantages varient d'un État à l'autre et peuvent inclure des limites sur la construction des équipements, une plus grande protection par rapport à l'annexion et l'expropriation, une éligibilité pour un programme d'achat de droit à bâtir et, souvent, une taxation préférentielle sur la propriété. Adaptable aux conditions locales, cet outil est flexible et plus efficace s'il est combiné au zonage agricole. L'inscription dans un district agricole, toutefois, est strictement volontaire, les avantages peuvent ne pas être assez attrayants pour les exploitants (surtout en zone périurbaine) et les sanctions liées au retrait du programme peuvent ne pas être suffisamment fortes pour empêcher la construction. Les districts agricoles peuvent encourager l'aménagement local, par exemple en limitant les autorisations de districts aux collectivités dotées de schémas spécifiques de protection des espaces agricoles et naturels.

Enfin, principalement décrétées au niveau des États, les lois sur le droit-àexploiter sont conçues pour protéger les exploitants agricoles contre les procès pour nuisance. Aujourd'hui, chaque État dispose d'une telle loi, affirmation politique de la valeur de l'agriculture. Certaines dispositions incluent une mention portée sur le titre des propriétés situées en zone agricole qui prévient les acheteurs de la possibilité de bruit, poussière, odeurs et autres inconvénients liés à l'activité agricole. Ces lois renforcent la position légale des agriculteurs vis-à-vis de nouveaux voisins nonagriculteurs et peuvent éduquer les résidents par rapport aux besoins de l'agriculture. Elles peuvent ne pas décourager l'engagement de poursuites pour nuisance. Cette disposition peut être rapprochée du régime de responsabilité civile français des «troubles anormaux de voisinage » qui aboutit sensiblement à la même solution (indemnisation des dommages de voisinage, entre un agriculteur et ses voisins, résultant de troubles considérés comme excessifs, le juge français pouvant prendre en compte la vocation agricole de la zone). Le système américain, toutefois, est spécifique aux activités agricoles, ce qui n'est pas le cas du régime français. De manière générale, les lois sur le droit-àexploiter n'ont pas été testées devant les tribunaux et il est donc difficile de savoir si elles peuvent aider à maintenir les espaces agricoles.

En conclusion, des outils présentés ci-dessus, le zonage de protection agricole, la taxation à valeur d'usage, l'achat de droit à bâtir et la loi sur le droit-à-exploiter sont les plus utilisés (AFT, 2002). Certaines communautés ont élaboré des programmes complets de protection des espaces agricoles et naturels en combinant zonage agricole, achat de droits à bâtir et district agricole : le zonage stabilise rapidement la base foncière et l'inscription dans un district aide à prévenir la conversion de surfaces importantes tandis que les exploitants attendent (parfois un certain temps) de vendre une servitude de protection permanente sur leur terrain. Environ $40 \%$ de la surface protégée par des programmes d'achat de droit à bâtir est soumise à une forme de zonage agricole (AFT, 2002).

Il convient de ne pas minimiser la volonté des américains de protéger leurs espaces agricoles et naturels. Ainsi, un recensement mené par le Land Trust Alliance (LTA, 2006) montre que sur la période 1998-2003, le nombre de land trusts a crû de 1213 à 1 $537(+27 \%)$ et la surface protégée par des conservation easements a progressé de 560490 ha à 2050918 ha (+266\%). D'autre part, une analyse du Trust for Public Land (TPL, 2006) montre que sur la période 19942005, $77 \%$ des mesures destinées à utiliser des fonds publics pour la conservation du foncier, soumises à référendum, ont été 
RECHERCHES

approuvées, générant une somme totale de 25,4 milliards d'euros. Situés en zones urbaines autant que rurales, les électeurs ont approuvé de telles mesures dans 45 États.

\section{Outils français de protection des espaces agricoles et naturels}

En termes d'intervention directe, l'État et les collectivités locales disposent de deux moyens principaux pour mener la politique foncière : le droit de préemption et la réserve foncière, qui permettent de lutter contre la spéculation et d'accommoder la croissance urbaine (Gry, 2002). Le droit de préemption est un outil souple qui peut être utilisé dans des situations spécifiques telles que la protection des espaces naturels sensibles ou plus généralement pour gérer des zones d'extension urbaine à vocation diverse. Les pouvoirs publics peuvent aussi créer des réserves foncières selon trois moyens d'acquisition principaux : l'acquisition à l'amiable, le droit de préemption et l'expropriation pour cause d'utilité publique. L'acquisition à l'amiable est la procédure préférée car la plus légère des trois ; la procédure d'expropriation est utilisée comme recours final lorsque les négociations ont échoué (Gry, 2002). Le zonage constitue également un moyen important de l'action foncière.

En France, les espaces agricoles et naturels bénéficient d'une protection particulière et de règles spécifiques d'utilisation du sol. La forêt fait l'objet de règles spécifiques (défrichement, par exemple) qui semblent difficiles à comparer au contexte américain. La forêt publique, d'ailleurs, constitue une protection efficace contre sa conversion vers l'urbain. Aux États-Unis, les forêts sous responsabilité du gouvernement fédéral font plutôt l'objet de débats quant à la construction de route et à leur exploitation sylvicole; plusieurs outils, comme l'achat et le transfert de droit à bâtir, sont utilisés pour protéger les espaces boisés. La présentation des outils français suit la typologie du tableau 2 et se base principalement sur Châteaureynaud $(2003)^{5}$.

Tout d'abord, les directives de développement incluent les deux schémas clés issus de la loi Solidarité et Renouvellement Urbains (SRU) en 2000 : le Schéma de Cohérence Territoriale (SCOT) et le Plan Local d'Urbanisme (PLU).

Le SCOT, innovation principale de la loi SRU (Gry, 2002), remplace le schéma directeur avec une fonction intégrative à l'échelle supracommunale (Casteigts, 2002). Parmi les documents d'un dossier de SCOT figure le projet d'aménagement et de développement durable. Ce document définit les grands projets d'équipements et de services, des objectifs quant à l'équilibre entre habitat, transport et commerces, et fixe des orientations générales de l'organisation de l'espace, en particulier les grands équilibres entre espaces urbains et à urbaniser et espaces naturels et forestiers. Le but d'un SCOT est d'assurer une cohérence de l'aménagement à des échelles variées et entre les politiques sectorielles dans une perspective de développement régional (Casteigts, 2002). Un SCOT est établi et approuvé par un Etablissement Public de Coopération Intercommunale (EPCI) ou un syndicat mixte, en association avec les services de l'État, après concertation avec le public.

Également créé par la loi SRU de l'an 2000, le PLU a remplacé le plan d'occupation des sols. Défini par la commune, en association avec les services de l'État, il est approuvé par le conseil municipal après enquête publique et modifications éventuelles. Tout comme un SCOT, un PLU comprend un projet d'aménagement et de développement durable qui formalise la vision stratégique du PLU : un plan pour l'ensemble de la commune avec des projets

5. Il faut aussi noter la publication du rapport Boisson (2005) sur la maîtrise foncière. 
détaillés par quartier et des engagements concrets en termes d'opérations d'aménagement. L'accent est mis sur le renouvellement urbain, la protection de l'environnement et la qualité architecturale. Le PLU définit quatre zones : urbaine (U), urbanisation future (AU), agricole (A) et naturelle (N). Il traite de tous les aspects du développement, y compris la destination des constructions, l'apparence extérieure des bâtiments, le tracé des voies et les secteurs à protéger.

SCOT et PLU ont pour objectif, entre autres, de protéger les espaces agricoles et naturels. En particulier, si une réduction importante des surfaces agricoles et forestières est prévue, les projets de SCOT et de PLU sont soumis à plusieurs organisations dont la commission départementale d'orientation de l'agriculture, la chambre d'agriculture, l'institut national des appellations d'origine et, le cas échéant, le centre régional de la propriété forestière. S'il n'y a pas de réponse de ces organisations sous deux mois après notification, leur avis est considéré favorable (Caillaud \& Aubert, 2003). La réduction des terres agricoles par un PLU, toutefois, ne constitue pas en soi une violation des règles d'aménagement.

Les dispositions de zonage incluent la règle de constructibilité limitée, les zones (A) et (N) du PLU et la zone agricole protégée.

Afin de lutter contre l'habitat dispersé, la règle de constructibilité limitée vise à interdire toute construction en dehors des parties actuellement urbanisées de la commune, à moins qu'un PLU (ou document d'urbanisme opposable) approuvé existe, condition de dévolution des pouvoirs d'aménagement au niveau local. Ainsi, il existe un paradoxe en ce sens que seules les communes choisissant de ne pas avoir de planification spatiale sont affectées par cette règle fondamentale. Ce principe a été assoupli grâce à des possibilités de dérogation
(Caillaud \& Aubert, 2003 ; Châteaureynaud, 2003) comme la possibilité d'une extension limitée qui nécessite l'accord du préfet et une étude d'impact sur l'extension urbaine et l'activité agricole. Le règlement national d'urbanisme stipule également que l'habitat ne doit pas être nuisible aux activités agricoles et forestières.

Les zones agricoles (A) sont précisément protégées dans le PLU pour leur potentiel agronomique, biologique ou économique. Les zones A sont exclusivement réservées à l'activité agricole ou biologique et comprennent les bâtiments et les installations qui sont nécessaires aux services publics et à l'activité agricole. La construction de bâtiments n'y est donc tolérée que si considérée comme indispensable à l'activité agricole. La loi SRU indique aussi des distances de séparation entre les bâtiments agricoles et les propriétés non agricoles.

Le PLU comprend également les zones (N) qui sont des zones naturelles ou forestières protégées pour la qualité du site ou du paysage et pour leur intérêt esthétique, historique ou écologique. Le développement peut y être autorisé si aucun dommage n'est porté à l'intérêt protégé, ou, à ce titre, déplacé via un transfert de COS (cf. infra). Enfin, l'élaboration du plan doit prendre en compte le document de gestion de l'espace agricole et forestier, notamment pour planifier l'urbanisation qui peut réduire de manière significative la surface de telles zones.

La zone agricole protégée a été créée pour lutter contre la fragmentation des terres agricoles à proximité des métropoles. Il s'agit d'une zone dont la protection présente un intérêt public en raison de sa localisation ou de la qualité de sa production. Cette zone soumet tout changement dans l'usage du sol, qui altère durablement le potentiel agricole, biologique ou économique de la zone, à l'approbation de la chambre d'agriculture et de la commis- 
RECHERCHES

sion départementale d'orientation de l'agriculture, à moins que le projet soit situé sur le territoire d'un PLU opposable. Les permis de construire sont également soumis à des conditions spécifiques. Enfin, cette zone, qui peut comprendre de petites aires boisées, peut être gérée par une association agricole pluricommunale pour promouvoir le regroupement des parcelles ou la continuité de l'activité agricole dans la zone.

Les outils d'acquisition de propriété font référence, à l'exception des transferts de Coefficient d'Occupation du Sol (COS), à l'usage du droit de préemption. Ils concernent ainsi les espaces naturels sensibles, les établissements publics fonciers locaux, les zones d'aménagement différé, le Conservatoire du littoral et les Sociétés d'Aménagement Foncier et d'Etablissement Rural (SAFER).

Les transferts de COS sont l'équivalent français des transferts de droit à bâtir américains. Le COS est le ratio entre la surface de plancher et la surface du terrain. La loi SRU applique les COS aux zones urbaines et d'urbanisation future seulement, et permet (de manière limitée) des transferts de COS dans les zones naturelles. Dans ce cas, deux valeurs de COS sont établies : l'une pour l'ensemble de la zone, identifiée par le PLU avec une valeur maximale de densité, l'autre pour une sous-zone spécifique. La zone émettrice perd ses droits de construction en raison d'une servitude administrative d'inconstructibilité, avec une valeur de COS moins élevée. En contrepartie, la zone réceptrice est bâtie à densité (COS) plus élevée en raison du regroupement des constructions, les droits à bâtir initiaux y étant augmentés de ceux liés au transfert. Le transfert de COS fait l'objet d'une procédure rigoureuse, dont la reconnaissance formelle de la servitude par un notaire (Caillaud \& Aubert, 2003). De plus, la valeur de COS augmentée (qui résulte du transfert) sur un terrain donné doit être compatible avec le schéma de cohérence territoriale (Châteaureynaud, 2003).
L'espace naturel sensible a été créé pour financer une politique de protection, de gestion et de développement limité des zones naturelles à des fins d'accès public. L'objectif est de protéger des zones naturelles sans limitation aux zones côtières et montagneuses. L'identification d'un tel espace implique un droit de préemption au bénéfice du département et pouvoir au préfet de prendre action pour la protection du site et du paysage en y limitant le développement (Châteaureynaud, 2003). La définition de l'espace naturel sensible peut être facilitée par une Zone Naturelle d'Intérêt Ecologique, Faunistique et Floristique (ZNIEFF) qui identifie des zones de patrimoine naturel. Une ZNIEFF n'a pas de statut légal mais peut servir de référence pour les autorités administratives.

Un établissement public foncier local peut être créé par le préfet pour contrôler l'usage du sol et éviter la spéculation foncière. En application de la loi SRU, cet outil permet aux établissements publics de coopération intercommunale de créer des réserves foncières en acquérant des terrains pour les municipalités membres (ou l'État), qui les rachètent en fonction de leurs besoins d'aménagement. Un tel établissement peut utiliser le droit de préemption et acquérir des terrains par expropriation.

Une zone d'aménagement différé peut être créée pour définir le périmètre des opérations d'aménagement futures et lutter contre la spéculation foncière en recourant au droit de préemption. Une telle zone peut ainsi servir à créer des réserves foncières. Le préfet peut créer une telle zone pour une durée maximale de 14 ans après suggestion par les communes intéressées. D'après la loi SRU, une zone d'aménagement différé doit être compatible avec un schéma de cohérence territoriale.

Le Conservatoire du littoral est un outil d'acquisition de propriété dont le fonctionnement est intéressant pour cette analyse. Etablissement public, le Conservatoire a 
pour mission de protéger les rivages maritimes et lacustres et d'assurer le respect des sites naturels et l'équilibre écologique. Le foncier acquis par le Conservatoire fait partie du domaine public et sa gestion peut être déléguée à une collectivité locale, un EPCI ou une association accréditée. L'activité principale du Conservatoire consiste à acquérir des biens pour un terme indéfini, soit à l'amiable, par expropriation pour cause d'utilité publique ou par préemption dans le cadre d'une zone d'aménagement différé ou des espaces naturels sensibles, ou par donation ou legs. En général, le Conservatoire cible ses acquisitions en fonction des orientations définies par le SCOT et le PLU. Aucun développement n'y est permis.

Les SAFER jouent un rôle important en matière de gestion des terres agricoles. Elles ont été créées en 1960 pour acheter des terres agricoles et les subdiviser afin d'aider l'agriculture familiale et faciliter l'installation des jeunes agriculteurs. Aujourd'hui, leur activité principale consiste à bâtir des réserves foncières et faciliter les échanges de terre afin de protéger les espaces ouverts (essentiellement agricoles). Disposant du droit de préemption, elles peuvent acquérir en priorité, dans certaines conditions, les propriétés agricoles mises en vente. L'acquisition amiable est cependant préférée, et le foncier est revendu sous 5 ans soit à des agriculteurs en activité qui augmentent leur surface d'exploitation, soit pour de nouvelles installations. Les SAFER peuvent remembrer les terres agricoles pour regrouper les petites propriétés en exploitations économiquement viables.

Enfin, les programmes d'incitation consistent en une taxation préférentielle du foncier non bâti et des politiques contractuelles. Par défaut, en France (et contrairement aux États-Unis), le foncier agricole est taxé à sa valeur d'usage courant, réduisant ainsi l'incitation à la conversion du foncier agricole et naturel non bâti. D'autre part, une politique contractuelle a été initiée entre le gouvernement et les exploitants agricoles sous la forme de Contrats Territoriaux d'Exploitation (CTE) mis en place en 1999. Les agriculteurs s'y engagent, en échange d'une aide financière et en fonction d'un cahier des charges, à respecter les paysages et l'environnement. Fin 2003, ils ont été remplacés par les Contrats d'Agriculture Durable (CAD), plus directifs en matière d'objectifs environnementaux et territorialisés pour mieux harmoniser les pratiques. En contrepartie d'une subvention de 27000 en moyenne pour cinq ans, l'exploitant promet, par exemple, de lutter contre la pollution de l'eau ou de planter des haies. Entre les CTE et les CAD, depuis 1999, plus de 35 000 contrats ont été signés.

En conclusion, la décentralisation s'est traduite par un pouvoir accru des maires par rapport à l'usage du sol, mais aussi par une fragmentation accrue des décisions d'usage du sol et un certain laxisme du contrôle de légalité de l'urbanisation (ReyLefebvre, 2004). Malgré cela, l'État continue de garder un contrôle sur la pratique de l'aménagement à travers la prescription du format des documents d'urbanisme (Cullingworth, 1994). Cependant, avec la réforme de l'intercommunalité, la plupart des communes françaises sont désormais membres d'un EPCI à fiscalité propre dont les compétences obligatoires incluent l'aménagement de l'espace. Ainsi, le contrôle technique de l'État via ses services déconcentrés a régressé. En pratique, vu que la majorité des communes sous pression d'urbanisation sont dotées d'un document d'urbanisme, la décentralisation a entraîné un net recul de l'influence de l'État sur l'usage du foncier.

Les politiques spécifiques françaises, en particulier par leur approche réglementaire, semblent offrir un ensemble de mesures de protection plus efficace qu'aux États-Unis. Peut-on tirer des enseignements de la comparaison de ces outils? 
RECHERCHES

\section{Des recommandations pour la protection des espaces agricoles et naturels français ?}

\section{Une synthèse des outils américains et français}

On a souligné plusieurs différences clés entre les systèmes d'aménagement français et américains, différences qui ont un impact sur les outils privilégiés en matière de protection des espaces agricoles et naturels : rôle limité du gouvernement fédéral aux États-Unis, au profit des collectivités locales et des États, alors qu'en France le gouvernement central continue de jouer un rôle (certes limité depuis la décentralisation); question de l'indemnisation des servitudes où les aménageurs américains font face aux tribunaux en cas de taking, ce qui tranche avec le principe de non-indemnisation des servitudes en France.

Alterman (1997) estime que les ÉtatsUnis connaissent, en général, un succès limité en matière de protection des terres agricoles, bien que les États dotés de plusieurs outils semblent plus efficaces. De manière plus surprenante peut-être, cet auteur évalue également comme faible le succès de la France en la matière, du moins par rapport à d'autres pays européens. La France protège mieux ses espaces agricoles que les États-Unis, mais ce résultat semble en grande partie dû à des normes de densités plus élevées.

Autre similitude : en dépit de systèmes d'aménagement différents, les deux pays présentent des outils dans les quatre catégories définies (directive de développement, zonage, acquisition de propriété, incitation). En ce sens, les deux pays sont au même stade de création d'une gamme diversifiée d'outils pour protéger leurs espaces agricoles et naturels.

En moyenne, cependant, le système français est probablement plus efficace que le système américain parce que plus cohérent sur l'ensemble du territoire. Avec une faible implication du gouvernement fédéral, les États-Unis ne peuvent garantir qu'un ensemble donné d'outils sera appliqué uniformément à travers le pays. En revanche, le fait que le gouvernement central joue un rôle (quoique limité en pratique) dans la planification spatiale garantit, par exemple, que la règle de développement limité existe partout en France. L'application de ce principe peut varier sur le terrain, mais son existence est néanmoins certaine. Un autre exemple est la taxation du foncier non bâti à sa valeur d'usage courant, qui est la règle par défaut en France, alors que les États américains doivent voter une loi spécifique en ce sens.

Les outils américains mettent davantage l'accent sur les approches par le marché alors que les outils français favorisent des approches réglementaires. Cela se constate, par exemple, dans la variété des schémas qui doivent être cohérents entre eux dans le système français : SCOT, PLU, sans mentionner les plans à l'initiative de l'État (e.g., directive territoriale d'aménagement), pour des milieux spécifiques (e.g., littoral) ou les plans sectoriels (e.g., schéma de service collectif). Les plans sont également soumis au contrôle de légalité, avec le rôle important du préfet comme garant de la cohérence et de la prise en compte des priorités de l'État au niveau local. Enfin, l'État et les collectivités locales disposent d'une marge de manœuvre plus importante pour la protection de l'environnement vu qu'ils n'ont pas à indemniser les propriétaires pour la perte du droit à bâtir.

Au contraire, les États-Unis tendent à favoriser une implication du gouvernement aussi limitée que possible, surtout en matière d'acquisition de foncier, tel qu'illustré par l'émergence des fiducies foncières privées ou des programmes de transfert de droit à bâtir. De plus, en raison de la question des takings, les outils les plus communément utilisés sont des programmes d'incitation (taxation préférentielle de la propriété) qui cherchent à influencer l'économie de la 
conversion des espaces agricoles et naturels, plutôt que des contrôles directs d'utilisation du foncier (Beesley, 1999).

En résumé, les États-Unis et la France ont créé un ensemble d'outils de protection des espaces agricoles et naturels, certes similaires dans la diversité des approches, mais différents dans leurs poids respectifs en termes d'adoption nationale vs. locale, ou d'approche réglementaire vs. marché.

\section{De nouveaux outils?}

Toutes les techniques présentées contribuent à protéger les espaces agricoles et naturels. La plupart des observateurs de l'usage du foncier estiment que les incitations fiscales sont l'outil de préservation le moins efficace, alors que des stratégies intégrées et complètes, qui combinent approches incitatives et réglementaires, sont les plus prometteuses pour une protection à long terme (AFT, 2002 ; Alterman, 1997 ; Beesley, 1999). Merlin (1995) estime, par ailleurs, que l'acquisition est la seule garantie absolue pour protéger le foncier de l'urbanisation.

La protection la plus efficace implique donc une combinaison d'outils adaptée à la situation politique locale, aux propriétaires, à l'économie des espaces visés et à la pression d'urbanisation (Daniels \& Bowers, 1997). Cette combinaison devrait viser un équilibre entre, d'une part, développement des ressources pour accommoder l'urbanisation et, d'autre part, protection de ces espaces.

Dans une perspective économique, Brueckner (2000) montre que l'étalement urbain est le résultat de trois forces principales : augmentation de la population, revenus croissants et diminution des coûts de navette domicile-travail (commuting costs). L'étalement urbain est alors le résultat de trois défaillances du marché correspondantes : défaillance de prise en compte des avantages des espaces naturels, navette excessive due à la défaillance de prise en compte des coûts sociaux de la congestion, et défaillance de prise en compte par le nouveau développement des coûts d'infrastructure qu'il engendre. Brueckner (2000) suggère des solutions pour contrer ces défaillances, solutions qui consistent à imposer des taxes sur le développement et un péage assumé par les navetteurs (commuters).

En outre, Alterman (1997) a listé cinq conditions pour une protection efficace des espaces agricoles (et naturels). D'abord, avec un déclin de la contribution de l'agriculture à l'économie locale, il existe un fossé grandissant entre protection de la campagne et protection de l'activité agricole, d'où la nécessité d'une redéfinition de la protection des espaces agricoles comme protection de la campagne. Ensuite, des politiques nationales guidées par l'aménagement du territoire et effectivement appliquées par les collectivités locales via une politique d'usage du sol forte, de façon à limiter l'urbanisation anarchique. Troisièmement, un accent mis sur la maîtrise de l'urbanisation par le renouvellement urbain, par des densités beaucoup plus élevées qu'aux États-Unis et par l'éducation à l'importance d'une bonne gestion du foncier pour les générations futures. Ensuite, des politiques de protection qui bénéficient d'un large appui de l'électorat. Enfin une limitation extrême des initiatives exurbaines.

En effet, un ensemble de techniques coordonnées constitue un facteur d'atténuation de la conversion des espaces agricoles et naturels, mais la volonté politique (et donc le soutien des populations locales) et les collaborations sont essentielles. Une coalition de protection peut inclure la société civile avec des groupes comme les clubs de sports en plein air ou les associations de résidents. Le contexte français souligne également la nécessité d'une coopération intercommunale véritable en la matière. 
RECHERCHES

Malgré le déclin de l'agriculture, les politiques actuelles reposent en grande partie sur des exploitants «jardiniers » du paysage pour entretenir l'espace ouvert. Le meilleur moyen de protéger les espaces agricoles et naturels, alors, n'est-il pas de maintenir la rentabilité de l'agriculture, de sorte que les exploitations restent en activité ? Les techniques de protection devraient servir à garantir qu'une surface critique est protégée (contiguïté), que cette protection est durable (long terme), que les prix du foncier restent abordables (installation et agrandissement des exploitations) et que les conflits avec le monde non agricole sont minimisés (Daniels \& Bowers, 1997). Outre les techniques décrites, des initiatives susceptibles d'améliorer la viabilité des exploitations comprennent la diversification des produits, la vente directe ou l'agritourisme.

Par ailleurs, Alterman (1997) avance plusieurs facteurs clés du succès des Pays-Bas en matière de protection des terres agricoles. D'abord, le système d'aménagement néerlandais repose sur une hiérarchie nationale-régionale-locale, avec des directives fortes émanant du gouvernement central. Ensuite, la protection des terres agricoles est explicitement justifiée sur la base de la protection des campagnes. Troisièmement, il existe un engagement fort en faveur de l'endiguement (containment) urbain et de densités élevées. Ensuite, il n'existe pas d'indemnisation en cas de refus de construction, avec une politique foncière communale qui suit la norme achat-viabilisation-revente : les collectivités achètent les terrains agricoles à leur valeur non bâtie, apportent les services publics et revendent le foncier viabilisé aux promoteurs en captant la plus-value.

On peut alors penser à six outils de protection des espaces agricoles et naturels qui pourraient compléter ceux existant en France. Dans la mesure où une évaluation des outils actuels ne constitue pas l'objet de cet article, ces propositions doivent être envisagées avec prudence et considérées comme des pistes à explorer pour alimenter le débat. Leur pertinence dépend des avantages potentiels « nets » (prenant en compte leurs aspects positifs et négatifs) par rapport aux objectifs que se fixe la société.

Une technique de protection des espaces émergente aux États-Unis est celle de l'arrêté d'atténuation (mitigation ordinance). Cette réglementation exige que pour chaque hectare agricole ou naturel converti à un autre usage, le promoteur protège une surface équivalente aux caractéristiques similaires sur le territoire de la même collectivité. Cette exigence peut être satisfaite en payant un droit à la collectivité ou en achetant des droits à bâtir aux exploitants. Outre les avantages liés au programme d'achat de droit à bâtir, cette technique assure une protection, quasiment hectare pour hectare, des espaces menacés. De plus, la technique pourrait s'appuyer sur le dispositif français de la servitude administrative d'inconstructibilité. D'autres outils américains innovants existent (voir Adelaja \& Schilling, 1999) mais reposent fortement sur une capacité à séparer aisément le droit de propriété du droit à bâtir, et semblent donc plus difficiles à transposer.

Ensuite, on pourrait, comme deuxième outil, étendre l'utilisation des transferts de COS des zones naturelles aux zones agricoles des plans locaux d'urbanisme. Aux États-Unis, cet outil est utilisé pour la protection des espaces agricoles et naturels, mais il est limité aux zones $\mathrm{N}$ en France. Pourquoi ne pas l'étendre aux espaces agricoles? Bien que théoriquement intéressant, cet outil n'a pas été appliqué de manière extensive pour plusieurs raisons (cf. supra). En France, aussi, les transferts de COS ont été appliqués de manière limitée et dans des situations différentes, ce qui rend une évaluation globale difficile (Renard, 1999). En particulier, des difficultés techniques, institutionnelles et éthiques ont été soulignées, difficultés qui limitent les applications pratiques des transferts de COS (Renard, 1999). Cependant, l'extension de cette 
méthode aux espaces agricoles augmenterait de fait le nombre d'applications. Une évaluation de ces applications, en France et outre-Atlantique, apporterait une plus grande compréhension des situations spécifiques dans lesquelles cette méthode est efficace.

Troisièmement, il semble que la seule garantie de non-urbanisation soit l'acquisition de droits à bâtir, à défaut l'acquisition de propriété. Le Conservatoire du littoral a déjà été présenté comme un outil d'acquisition de propriété actuellement limité aux rivages maritimes et lacustres. Pourquoi ne pas étendre la mission du Conservatoire aux zones agricoles et naturelles des PLU (quitte à rebaptiser ledit Conservatoire), ou bien créer un établissement public similaire au Conservatoire dont la mission serait ciblée sur ces zones ? Ces espaces bénéficieraient ainsi d'un programme d'acquisition les protégeant à perpétuité. Vue la portée potentielle de cette recommandation, toutefois, il serait important de restreindre un tel programme aux zones A et $\mathrm{N}$ présentant des caractéristiques paysagères spéciales ou une valeur écologique unique. Le principal frein à la mise en application de cet outil serait le volume des fonds requis pour l'acquisition de terrains. Une autre possibilité serait que cet organisme public n'ait pas pour vocation l'acquisition de propriété mais celle de droits à bâtir, solution beaucoup moins coûteuse. Cela consisterait à généraliser le dispositif évoqué en note 3 de cet article : servitude conventionnelle où le propriétaire privé est indemnisé pour l'interdiction de bâtir pesant sur son terrain, c'est-à-dire une solution compatible avec le droit (servitude) et de gestion plus simple (droit d'usage maintenu pour le propriétaire).

Par conséquent, un quatrième outil servant à résoudre en partie les problèmes de financement serait de transférer la politique néerlandaise de revente des terrains agricoles viabilisés aux promoteurs (via le droit de préemption, par exemple). De cette façon, la collectivité dans son ensemble capterait la plus-value du foncier liée à la pression d'urbanisation. Bien entendu, il est possible d'imaginer que cette plus-value soit davantage répartie entre les acteurs de l'aménagement, dont les propriétaires des terrains. Les sommes générées pourraient être placées dans un fonds spécial utilisé par les collectivités pour l'acquisition de foncier. Les fonds pourraient aussi être attribués sur la base d'un concours entre propriétaires pour des projets de protection spécifiques, ou alloués à des espaces remarquables sur la base de priorités déterminées en association entre État, collectivités locales et société civile. Suivant Brueckner (2000), il est également possible d'envisager une taxe qui récupèrerait les plusvalues d'urbanisation. Liée à la pression d'urbanisation et peut-être plus simple à mettre en place que le recours au droit de préemption, cette taxe, payée par les promoteurs, freinerait d'autant le processus de conversion des terrains non bâtis, et pourrait alimenter le fonds d'acquisition évoqué.

$\mathrm{Si}$ les propositions trois et quatre vont dans le sens d'achats fonciers (ou de droits à bâtir) par les pouvoirs publics, elles posent les questions de la mise en valeur de ces terres et de l'extension du domaine public. En effet, le statut du fermage actuel n'est pas très bien adapté et une mise en valeur directe par l'État ou des collectivités semble difficile à envisager, alors que les politiques actuelles tendent à la privatisation du domaine public. L'enjeu de la mise en valeur est lié à celui de l'usage des terres. Si l'usage est agricole ou forestier privé, alors une politique de contractualisation (proposition 5) pourrait faciliter le maintien de cet usage dans le temps ; la solution de la servitude conventionnelle simplifie également cette question. Quant à la tendance à la privatisation, elle est en partie la conséquence du manque de moyens affiché par les pouvoirs publics. La proposition 4, relative au financement des acquisitions par la captation de la plus-value d'urbanisation, limiterait l'argument financier à propos du manque de 
RECHERCHES

réserves foncières, un des pivots de la mâ̂trise de l'espace.

Un cinquième outil serait la promotion de politiques contractuelles, qui semblent trouver un équilibre entre approches basées sur le marché et approches réglementaires. Les contrats permettent aux exploitants ou aux propriétaires fonciers et aux collectivités locales d'identifier des situations gagnantgagnant. Cela tend à mettre en valeur la nature multifonctionnelle de l'agriculture et de la sylviculture, avec productions et services fournis à la communauté en échange d'une compensation spécifique. Les contrats peuvent aussi servir à minimiser les conflits potentiels entre exploitants et non-exploitants, et donc à accroître la durabilité des exploitations dans les zones périurbaines.

Enfin, sixième outil, il s'agirait de créer des zones spécifiques pour la protection des espaces agricoles et naturels dans les zones périurbaines, en permettant l'acquisition de foncier par les collectivités locales, établissements publics fonciers locaux ou autres organisations accréditées. A l'intérieur de périmètres délimités en fonction de la valeur productive et paysagère des terrains, les acquisitions pourraient être réalisées à l'amiable, par préemption ou par expropriation. Les terrains acquis pourraient être donnés ou loués en fonction d'objectifs de protection. Une proposition similaire a été récemment adoptée dans le cadre de la loi relative au développement des territoires ruraux (loi n²005-157 du 23 février 2005) en accordant aux départements le droit de délimiter des périmètres d'intervention et d'acquérir des terrains pour protéger et mettre en valeur des espaces agricoles et naturels périurbains.

Si cette loi va effectivement dans le sens de la sixième proposition, on peut regretter que son application prenne du temps : le décret en Conseil d'État qui détermine les conditions d'application du chapitre portant sur la protection des espaces agricoles et naturels périurbains est, début juin 2006, en attente de publication. De plus, même si la loi mentionne l'accord nécessaire avec les autres collectivités intéressées (communes, établissements publics compétents en matière de plan local d'urbanisme), on peut regretter que la loi place le département au centre de la délimitation de ces périmètres d'intervention. En effet, de par son échelle, le département n'a pas vocation à intervenir spécifiquement sur les espaces périurbains (à moins que ces derniers recoupent les espaces naturels sensibles). On aurait pu souhaiter que la délimitation de zones de protection des espaces agricoles et naturels périurbains repose davantage sur les communes dépassant un certain seuil de population ou, mieux, aux établissements publics de coopération intercommunale qui ont pour vocation centrale l'aménagement de l'espace à l'échelle pluricommunale.

En conclusion, les propositions décrites dans cet article pourraient être appliquées à la protection des espaces agricoles et naturels présentant une valeur productive, esthétique, paysagère, écologique ou récréative. La mise en œuvre et l'efficacité de ces mesures dépendent non seulement des efforts coordonnés de tous les niveaux de gouvernements, des propriétaires fonciers et autres acteurs de l'aménagement, mais aussi de l'intégration des enjeux économiques, sociaux et environnementaux dans des programmes de protection complets. Ce ne sont pas seulement les espaces agricoles et naturels qui sont en jeu, mais aussi une meilleure compréhension de l'impact de la destination des sols sur la qualité de vie locale.

* L'auteur remercie les deux referees pour leurs riches commentaires. Une version antérieure de cet article a été présentée au congrès annuel de l'Association of European Schools Of Planning, Grenoble (France), 1-4 juillet 2004. Cet article a été soumis lorsque l'auteur était à I'Institut Supérieur d'Agriculture de Lille, remercié pour son soutien financier. 


\section{RÉFÉRENCES BIBLIOGRAPHIQUES}

Adelaja A.-O. ; Schilling B.-J. Innovative approaches to farmland preservation In The rural-urban fringe in North America, Furuseth O.-J. \& Lapping M.-B. (eds.), Contested countryside Ashgate, Aldershot, UK, 1999, pp. 113-135.

AFT (American Farmland Trust). Why save farmland? Disponible en ligne <www.farmlandinfo.org/documents/ 28562/FS_Why Save Farmland_1-03 .pdf>, 2003.

AFT (American Farmland Trust). The farmland protection toolbox, disponible en ligne <www.farmlandinfo.org/documents/27761/FS_Toolbox_10-02.pdf >, 2002.

AFT (American Farmland Trust). Saving American farmland: What works, AFT publications, Northampton, MA, 1997.

Agreste. L'utilisation du territoire en 2003 : Nouvelle série 1992 à 2003, Ministère de l'Agriculture, Service central des enquêtes et études statistiques, Chiffres et données agriculture, 2004, mars, ${ }^{\circ} 157$.

Alterman R. The challenge of farmland preservation : Lessons from a six-nation comparison, Journal of the American Planning Association, 1997, vol. 63, n 2 , pp. 220-243.

Beesley K.-B. Agricultural land preservation in North America : A review and survey of expert opinion In The ruralurban fringe in North America, Furuseth O.J. \& Lapping M.B. (eds.), Contested countryside, Ashgate, Aldershot, UK, 1999, pp. 57-91.

Boisson J.-P. La maîtrise foncière : Clé $d u$ développement rural, avis et rapports du Conseil Economique et Social, ${ }^{\circ}$ 5, 2005.

Brabec E. The economics of preserving open space, In Rural by design : Maintaining small town character, Arendt R. (ed.),
APA Planners Press, Chicago, IL, 1994, pp. 280-288.

Brueckner J.-K. Urban sprawl : Diagnosis and remedies, International Regional Science Review, 2000, vol. 23, n 2, pp. 160-171.

Caillaud M. ; Aubert B. La loi SRU en 90 questions, Le Moniteur, Paris, France, 2003.

Casteigts M. L'aménagement du territoire et l'environnement In Les collectivités locales en France, La Documentation Française, Paris, France, 2002, pp. 112116.

Cavailhès J. Espaces ruraux et espaces agricoles en France : Quelle mise en valeur du territoire? In Déméter 96 : Economie et stratégies agricoles, Armand Colin, Paris, 1995, pp. 129-180.

Châteaureynaud P. Dictionnaire de l'urbanisme, Le Moniteur, Paris, 2003.

CIA (Central Intelligence Agency). The world factbook, disponible en ligne $<$ www.cia.gov/cia/publications/factbook/index.html>, 2004.

Cullingworth J. B. Alternate planning systems : Is there anything to learn from abroad? Journal of the American Planning Association, 1994, vol. 60, $\mathrm{n}^{\circ} 2$, pp. 162-172.

Daniels T. A cautionary reply for farmland preservation. Planning \& Markets, 1999a, vol. 2, $\mathrm{n}^{\circ}$ 1, <www-pam.usc.edu/volume2 /v2i1a4print.html>

Daniels T. When city and country collide : Managing growth in the metropolitan fringe, Island Press, Washington, DC, 1999b.

Daniels T. ; Bowers D. Holding our ground: Protecting America's farms and farmland, Island Press, Washington, DC, 1997. 
Gordon P. ; Richardson H.W. Farmland preservation and ecological footprints: Acritique, Planning \& Markets, 1998, vol.1, $\mathrm{n}^{\circ} 1$, <wwwpam.usc.edu/volume1/v1i1a2 print.html >

Gry Y. Urbanisme et politique foncière In Les collectivités locales en France, La Documentation Française, Paris, France, 2002, pp. 122-127.

LTA (Land Trust Alliance). National land trust census, disponible en ligne <www.lta.org/census>, 2006

Merlin P. Les techniques de l'urbanisme, Presses Universitaires de France, Paris, 1995.
NASS (National Agricultural Statistics Service). Farms and land in farms: Final estimates by state and United States, U.S. Department of Agriculture, Agricultural statistics board, Statistical bulletins 19882002, <usda.mannlib.cornell.edu>, 2004.

Renard V. Où en est le système des transferts de COS ? Etudes Foncières, 1999, $\mathrm{n}^{\circ} 82$, pp. 8-16.

Rey-Lefebvre I. Face à la spéculation foncière, les maires sont trop souvent attentistes, Le Monde, 2 juin, <www.lemonde.fr>, 2004.

TPL (the Trust for Public Land). LandVote database, disponible en ligne <www.tpl.org>, 2006. 